\title{
APLICACIÓN DE LA TÉCNICA QUIRÚRGICA DE TRABECULECTOMÍA PARA EL TRATAMIENTO DEL AUMENTO DE LA PRESIÓN INTRAOCULAR ENCANINOS
}

\author{
Juan Carlos Lau-Choleón G. ${ }^{1}$, Víctor Fernández A. ${ }^{2}$, Diego Díaz C. ${ }^{2}$ y \\ Julio Olano Vargas ${ }^{3}$
}

\section{A Bstract}

The present study is an adjustment of the surgical technique of trabeculectomy for the treatment of the canine glaucoma. Five dogs of varios breeds, age and sex with clinical history of glaucoma were used. The surgical technique of trabeculectomy facilitated a new path for the drainage of the aqueous humor. The results indicate that the trabeculectomy is en easy and reliable option for the resolution of the high introcular pressure in dogs.

Key words: trabeculectomy, glaucoma, canine

\section{Resumen}

El presente trabajo es una adaptación de la técnica quirúrgica de trabeculectomía para el tratamiento del glaucoma canino. Para tal fin se utilizaron 5 caninos de distintos sexos, razas y edades, y con una historia clínica de glaucoma. Se empleó la técnica quirúrgica de trabeculectomía, la cual consiste en crear una nueva vía de drenaje del humor acuoso. Los hallazgos mostraron en todos los casos una importante disminución de la presión intraocular $(\mathrm{P}<0.01)$. Se demostró que la trabeculectomía es una alternativa, rápida y confiable para el tratamiento resolutivo de la presión intraocular en caninos.

Palabras clave: trabeculectomía, glaucoma, caninos

\section{INTRODUCCión}

El glaucoma es el aumento de la presión intraocular por encima de la presión compatible con la función normal. Está incluido en el grupo de enfermedades que manifiestan en común un nivel incrementado de presión intraocular originando daño tisular por lesiones en el nervio óptico (Morgan, 1999). En la mayoría de los casos el glaucoma es causado por obstrucción o estenosis de las vías de salida del humor acuoso provocando deterioro de la visión. El glaucoma es una de las causas más frecuentes de ceguera en el canino (Slatter, 1992).

La trabeculectomía es una técnica quirúrgica utilizada ampliamente en humanos para disminuir la presión intraocular (Boyd, 1993). Por tal motivo, el objetivo del presente trabajo fue evaluar esta técnica en la resolución del aumento de la presión intraocular observada en el glaucoma canino.

\footnotetext{
${ }^{1}$ Práctica privada

${ }^{2}$ Clínica de Animales Menores, UNMSM-FMV. E-mail: vfernandeza@vet.unmsm.edu.pe

${ }^{3}$ Hospital Daniel Alcides Carrión, Lima
} 


\section{Materiales y Métodos}

\section{Lugar y Animales}

El presente estudio se llevó a cabo en la Clínica de Animales Menores de la Facultad de Medicina Veterinaria de la Universidad Nacional Mayor de San Marcos. Para la ejecución del presente estudio se utilizaron 5 caninos de diferentes sexos, razas y edades, que en la evaluación clínica oftalmológica resultaron con diagnóstico definitivo de glaucoma. Los casos fueron registrados entre los meses de febrero del 2000 hasta mayo del 2001.

\section{Preparación del Paciente}

\section{a) Premedicación}

Se suministró antibióticos de amplio espectro (cloranfenicol al $1 \%$ ), por un lapso de 3 días antes de la intervención quirúrgica a fin de reducir la posibilidad de infección postoperatoria (Severin, 1991). Además, se administró timolol al $0.5 \%$ durante el mismo periodo para reducir la producción del humor acuoso (Wilkie y Latimer, 1991).

\section{b) Preparación prequirúrgica}

Los pacientes se prepararon de la misma manera que para cualquier otro procedimiento quirúrgico oftalmológico. Se utilizó un tranquilizante por vía endovenosa (maleato de acepromacina al $1 \%$ ).

Se limpió profundamente la piel con solución fisiológica estéril y se desinfectaron los párpados con una solución diluida de iodóforo (Severin, 1991). Se administraron gotas anestésicas oftálmicas (propacaína al $0.5 \%$ ) y posteriormente ofloxacino al $5 \%$ (Cator et al., 2001). Asimismo, se administró monitol al $20 \%$ vía endovenosa para disminuir la presión intraocular del ojo a intervenir (Dugan et al., 1989; Herrera, 1998).

\section{c) Anestesia}

Para realizar éste procedimiento quirúrgico se utilizó anestesia general (Ketamina al 50\% por vía endovenosa) (Severin, 1991; Magrane, 1971).

\section{Método Quirúrgico}

La fijación de los párpados se hizo con un espéculo de alambre. Se cubrió al paciente con paños y campos para cirugía ocular. La técnica quirúrgica fue como sigue (Boyd, 1993):

- Se hizo un colgajo conjuntival con base fórnix de $10 \mathrm{~mm}$ de longitud en el limbo con una tijera de mayo pequeña. Se hizo una disección atraumática entre la conjuntiva, la epiesclera y la esclera hasta 5 mm detrás del limbo (Fig. 1).

- La superficie escleral se limpió y se realizó un colgajo escleral de $3 \mathrm{~mm} \times 3 \mathrm{~mm}$ en el área desnuda de la esclera. Estos cortes se unieron por una incisión de 3 $\mathrm{mm}$ de longitud en el limbo, lo cual asegura que las líneas de sutura conjuntival y escleral se encuentren separadas (Fig. 2). El grosor del colgajo escleral se estimó por las incisiones posteriores, permitiendo un colgajo escleral de igual grosor. El colgajo es la mitad del grosor de la esclera.

- Se procedió a delinear con bisturí un triángulo equilátero de superficie y de trabéculo de $2 \mathrm{~mm}$ de lado, en la esclera profunda al colgajo escleral, extendiéndose anteriormente al limbo quirúrgico (Fig. 3).

- Se realizó la incisión anterior al limbo, extendiéndose hacia la cámara anterior. Con una tijera de Vannas se disecó cuidadosamente y la incisión anterior se completó extendiéndose a lo largo de los lados sin perder la cámara anterior. Este 
colgajo se removió por una incisión posterior justo en el tejido trabecular, el cual comenzó a ser dirigido hacia atrás con las pinzas (Fig. 4).

- Con una pinza de iris se ingresa a la cámara anterior y se extrae cuidadosamente una pequeña porción de iris por la ventana escleral (Fig. 5).

- Se efectuó una iridectomía a través de la ventana escleral (Fig. 6).

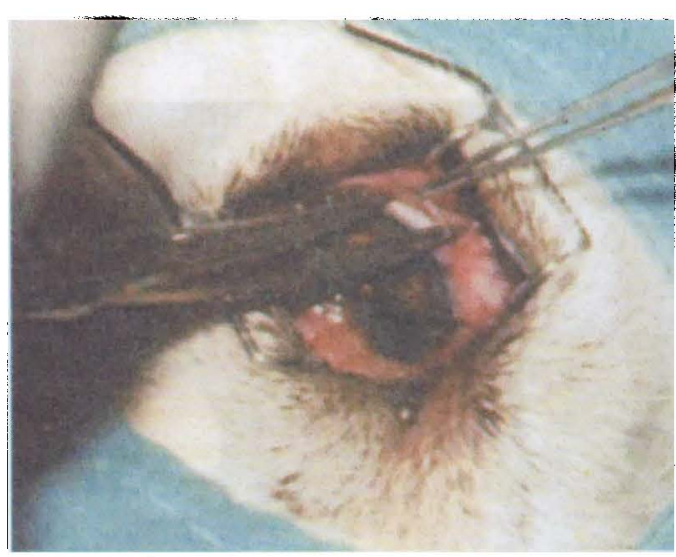

Fig. 1. Disección del colgajo conjuntival

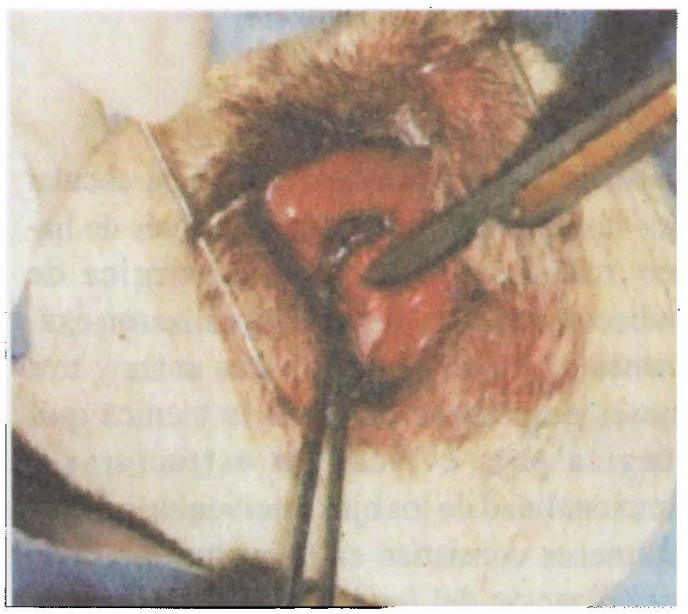

Fig. 3. Incisión del colgajo escleral profundo
- Una vez que el colgajo escleral externo de grosor parcial se recolocó dentro del lecho escleral, se hicieron de 2 a 4 suturas de nylon o vicryl 8-0 en el colgajo escleral (Fig. 7).

- Posteriormente se efectuó el cierre conjuntival con suturas adicionales de nylon o vicryl 8-0 (Fig. 8).

- Al retirar el blefarostato, se logró apreciar la formación de una vesícula filtrante.

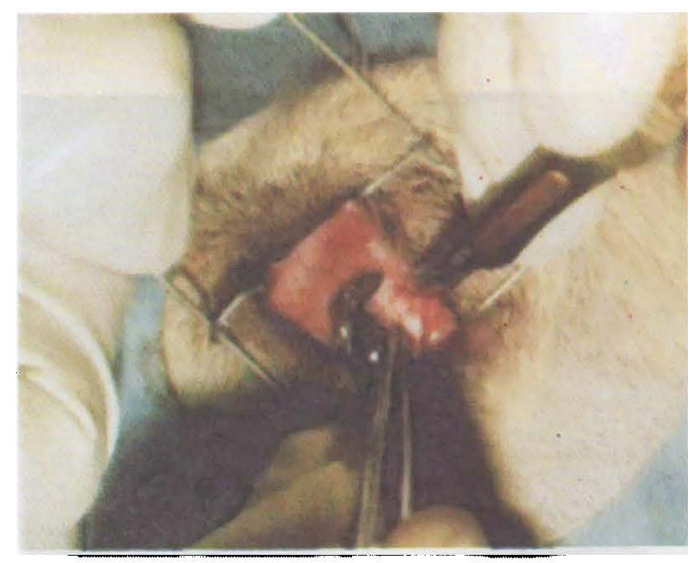

Fig. 2. Incisión del colgajo escleral superficial

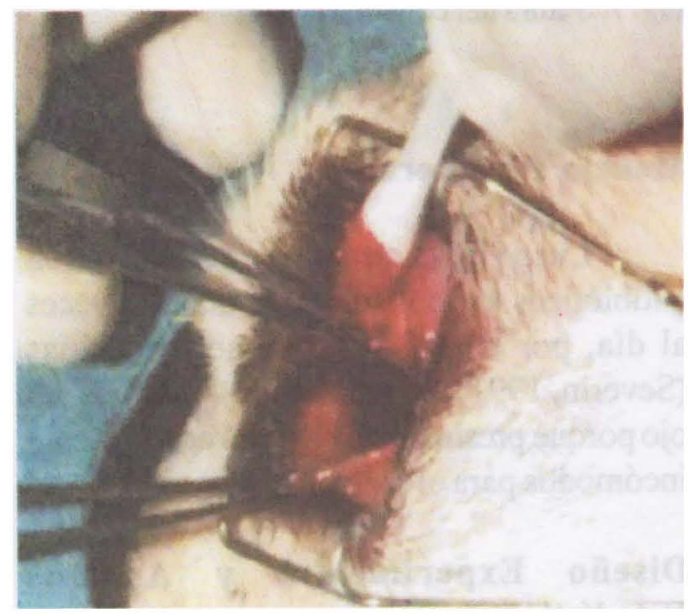

Fig. 4. Extracción del colgajo escleral profundo (trabeculectomía) 


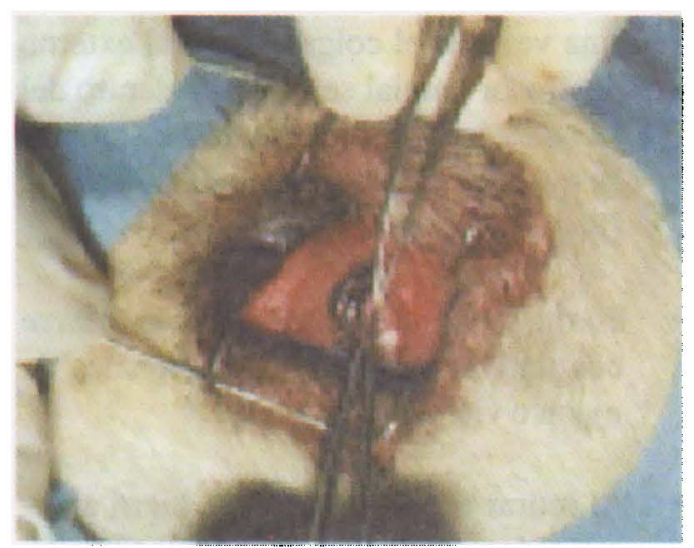

Fig. 5. Ingreso a la cámara anterior

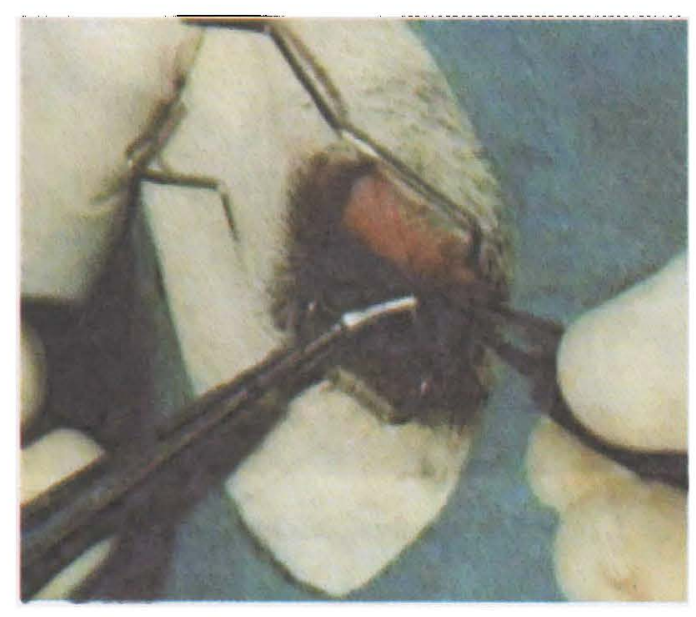

Fig. 7. Sutura del colgajo escleral

\section{Manejo Postoperatorio}

Luego de la cirugía, se administraron antibióticos tópicos (cloranfenicol), 3 veces al día, por un lapso no menor de 7 días (Severin, 1991). Se evitaron los vendajes del ojo porque presionaban el globo ocular y eran incómodos para el animal.

\section{Diseño Experimental y Análisis Estadístico}

El diseño metodológico se basó en el seguimiento secuencial de cada paciente durante el tiempo de recuperación postoperatorio y el registro individual de cada uno.

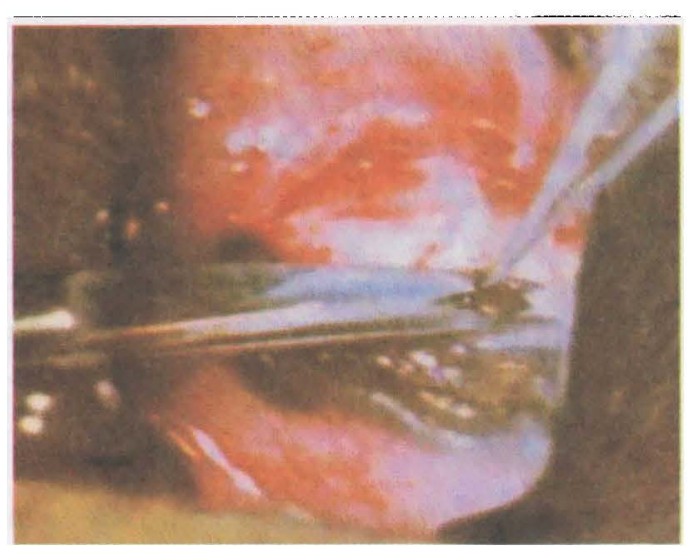

Fig. 6. Ejecución de la iridectomía

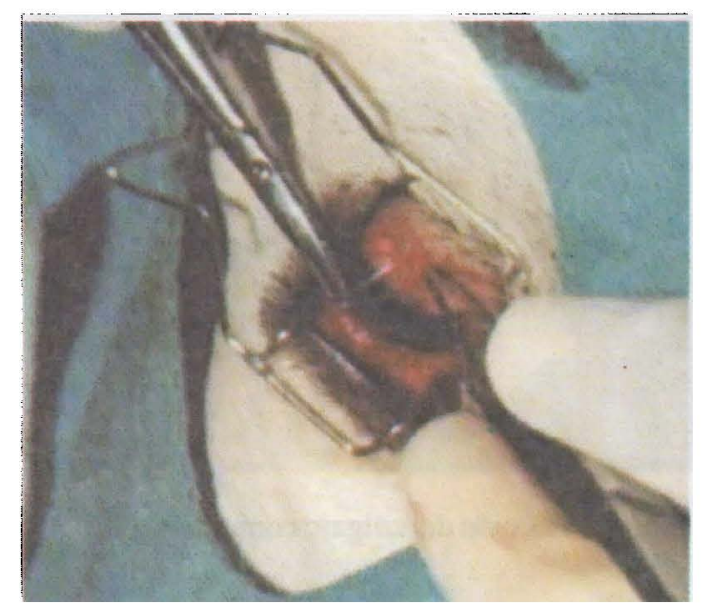

Fig. 8. Sutura de la conjuntiva

Se realizó la medición de la presión intraocular dos horas antes y tres meses después de haber realizado la técnica quirúrgica de trabeculectomía. Además, se realizaron exámenes oftalmológicos dos días antes y tres meses después de realizada la técnica quirúrgica para evaluar las estructuras y funcionalidad de los ojos intervenidos. Estos exámenes consistían en la evaluación de la visualización del fondo de ojo, ausencia de edema de la córnea y contracción de la pupila.

Para el análisis estadístico se utilizó la prueba de " $t$ " de Student para variables pareadas dependientes. 


\section{Resultados}

En la evaluación previa a la intervención quirúrgica, el paciente $\mathrm{N}^{\circ} 5$ presentó fondo de ojo "ausente", es decir, no se lograron observar las estructuras oculares internas. En todos los casos se apreció edema corneal, aunque de menor gravedad en los pacientes $\mathrm{N}^{\circ} 1$ y $\mathrm{N}^{\circ} 2$. Se observó midriasis en todos los casos (Cuadro 1).

La presión intraocular de los ojos glaucomatosos de los pacientes a los que se les realizó la técnica quirúrgica de trabeculectomía presentaron disminución de sus valores patológicos a valores normales después de 3 meses de realizada la técnica quirúrgica (Cuadro 2).

Ninguno de los pacientes presentó complicaciones postquirúrgicas. Luego de tres meses, se pudo observar las estructuras del fondo de ojo, el diámetro pupilar y la estructura corneal en condiciones normales, excepto en el paciente $\mathrm{N}^{\circ} 3$, que presentó un leve edema corneal (Cuadro 1).

Cuadro 1. Evaluación del fondo de ojo, córnea y pupila antes de la intervención quirúrgica

\begin{tabular}{ccccccc}
\hline \multirow{2}{*}{ Paciente } & \multicolumn{2}{c}{ Fondo de Ojo } & \multicolumn{2}{c}{ Córnea } & \multicolumn{2}{c}{ Pupila } \\
& Antes & Después & Antes & Después & Antes & Después \\
\hline 1 & P & P & + & - & M & - \\
2 & P & P & + & - & M & - \\
3 & P & P & ++ & + & M & - \\
4 & P & P & ++ & - & M & - \\
5 & A & P & ++ & - & M & - \\
\hline
\end{tabular}

A: Ausente; P: Presente; -: Normal; +: Edema leve; ++: Edema moderado; M: Midriasis

Cuadro 2. Diferencias de la presión intraocular $(\mathrm{mm} \mathrm{Hg})$ en caninos, dos horas antes y tres horas después de realizar la técnica quirúrgica de trabeculectomía

\begin{tabular}{cccc}
\hline \multirow{2}{*}{ Paciente } & \multicolumn{2}{c}{ Presión Intraocular } & Diferencia \\
\cline { 2 - 3 } & Pre operatoria & Post operatoria & \\
\hline 1 & 34.5 & 18.6 & $15.9(\mathrm{p}<0.01)$ \\
2 & 55.0 & 24.2 & $30.8(\mathrm{p}<0.01)$ \\
3 & 78.6 & 28.0 & $50.6(\mathrm{p}<0.01)$ \\
4 & 43.3 & 23.8 & $19.5(\mathrm{p}<0.01)$ \\
5 & 68.0 & 26.0 & $42.5(\mathrm{p}<0.01)$ \\
\hline
\end{tabular}




\section{Discusión}

Los animales en estudio presentaron uno o varios de los signos de la patología estudiada, los cuales fueron corroborados mediante la tonometría instrumental para el diagnóstico definitivo del glaucoma (Magrane, 1971; Slatter, 1992; Morgan, 1999; PetersonJones y Crispin, 1999).

Los valores obtenidos en los ojos de los pacientes con diagnóstico de glaucoma que fueron intervenidos quirúrgicamente, se encontraron por encima del rango normal de valores de presión intraocular (Magrane, 1971; Munger, 1998; Morgan, 1999; Severin, 1991; Peterson-Jones y Crispin, 1999).

Para el procedimiento quirúrgico de trabeculectomía, cabe resaltar la importancia de la anestesia tópica de la córnea antes de proceder a la cirugía oftalmológica. La solución de propacaína al $0.5 \%$ es una de las más usadas y brinda suficiente analgesia. Por otro lado, la anestesia general para los procedimientos quirúrgicos oftalmológicos es la que mejores resultados ha brindado (Magrane,1971).

En la técnica quirúrgica cabe, resaltar el colgajo escleral con base limbo para lograr una mejor exposición y visualización del campo operatorio, facilitando la disección del colgajo escleral en la córnea (Boyd, 1993).

El cuadro clínico de los pacientes glaucomatosos evolucionó a valores clínicos normales, sin importar la presión intraocular elevada que había antes de la cirugía.

Todos los animales presentaron edema corneal antes de realizar la técnica quirúrgica, concordando con lo señalado por Slatter (1992). El paciente $\mathrm{N}^{\circ} 3$ fue al único que se le encontró un edema moderado en la córnea y que luego de la cirugía llegó a mantener un edema leve. Sin embargo, los otros signos clínicos del glaucoma desaparecieron.

\section{Conclusiones}

- La técnica quirúrgica de trabeculectomía es sencilla y constituye una nueva alternativa confiable y muy útil para la resolución del glaucoma canino.

- La presión intraocular disminuyó a valores clínicamente normales dentro de los tres meses de realizada la intervención quirúrgica.

\section{LTERATURA CTtada}

1. Boyd, B. 1993. Highlights of ophthalmology. Word Atlas. Tomo 2. p 202-211. Ed. Interamericana. Panamá.

2. Cator, L; LE. Donnepleld; L Ketz; W. Gee; C. Finley; V. Lakhani; J. Hopo; K. Flarty. 2001. Penetración de ofloxacina y de ciprofloxacina en el humor acuoso de ojos con blef de filtración. Arch. Ophthalmology USA 119: 1117-1119.

3. Dugan, S.J.; S.M. Roberts; G.A. Severin. 1989. Systemic osmotherapy for ophthalmic disease in dogs and cats. J.A.V.M.A. 194: 115-118.

4. Herrera, D. 1998. Tratamiento quirúrgico del glaucoma canino con gonioimplantes simplificados no valvulados. Ophthalmology presentations. ISVO / SOLOVE AND WSAVA / AVEACA CONGRESSES in Buenos Aires. Argentina.

5. Magrane, W. 1971. Canine ophthalmology. $2^{\mathrm{a}}$ ed. p 181-214. Ed. Lea \& Febiger. Philadelphia, USA.

6. Morgan, $R$. 1999. Clínica de pequeños animales. $3^{a}$ ed. p 1030-1035. Ed. Harcourt Brace. Madrid, España.

7. Munger, R.J. 1998. Creando su práctica en oftalmología: "Hay que saber cuándo hay que tirar las cartas; y cuándo tenerlas en la mano". Ophthalmology presentations ISVO / SOLOVE AND WSAVA / AVEACA CONGRESSES in Buenos Aires. Argentina. 
8. Peterson-Jones, S.M.; S. Crispin. 1999. Manual de oftalmología en pequeños animales. $2^{a}$ ed. p 181-214. Ed. Romany Walls. España.

9. Severin, G. 1991. Manual de oftalmología veterinaria. $2^{\mathrm{a}}$ ed. p 185-190. Ed. Hemisferio Sur. Buenos Aires, Argentina.
10. Slatter, D. 1992. Fundamentos de oftalmología veterinaria. $2^{a}$ ed. $\mathrm{p}$ 397-428. Ed. Intermedia. Buenos Aires, Argentina.

11. Wilkie, D.A.; C.A. Latimer. 1991. Effect of topical administration of timolol maleate on intraocular pressure and pupil size in dogs. Am. J. Vet. Res. 52: 432-435. 